Article

\title{
Morphology and Thermal Properties of Calcium Alginate/Reduced Graphene Oxide Composites
}

\author{
Wanting Zhao ${ }^{1}$, Yan $\mathrm{Qi}^{1}{ }^{1}$, Yue Wang ${ }^{1}$, Yun Xue ${ }^{1}$, Peng $\mathrm{Xu}^{1}$, Zichao $\mathrm{Li}^{2, *(\mathbb{D})}$ and Qun $\mathrm{Li}^{1}{ }^{1, *(1)}$ \\ 1 College of Chemistry and Chemical Engineering, Qingdao University, Qingdao 266071, China; \\ 2016020406@qdu.edu.cn (W.Z.); qiyanqdu@126.com (Y.Q.); wangyue@qdu.edu.cn (Y.W.); \\ xueyun@qdu.edu.cn (Y.X.); 2017020860@qdu.edu.cn (P.X.) \\ 2 College of Life Sciences, Qingdao University, Qingdao 266071, China \\ * Correspondence: zichaoli@qdu.edu.cn (Z.L.); qunli@qdu.edu.cn (Q.L.); Tel.: +86-532-8595-0705 (Q.L.)
}

Received: 21 August 2018; Accepted: 4 September 2018; Published: 5 September 2018

\begin{abstract}
Calcium alginate ( $\mathrm{CaAlg}$ ) is a kind of biodegradable and eco-friendly functional material, and $\mathrm{CaAlg} /$ reduced graphene oxide (rGO) composites are expected to be applied as new textile, heat-generating, and flame-retardant materials. In this paper, the $\mathrm{CaAlg} / \mathrm{rGO}$ composites were prepared by a sol-gel method and their morphological and thermal properties were studied. The results showed that the introduction of rGO can efficiently improve the thermal stability of CaAlg. Further study showed that rGO increased the carbon formation rate by $4.1 \%$, indicating that the thermal stability was improved by the promotion of carbon formation. Moreover, the weight loss rate of the composites was faster at $180-200{ }^{\circ} \mathrm{C}$ than that of $\mathrm{CaAlg}$, after which the rate was less comparatively, suggesting the better thermal stability of the composite. This maybe because the high heat transfer efficiency of rGO allowed the material to reach the temperature of the thermal decomposition of the glycan molecule chain within a short time, and then promoted carbon formation. The thermal cracking mechanism of the composites is proposed based on the experimental data.
\end{abstract}

Keywords: calcium alginate; reduced graphene oxide; composites; morphology; thermal stability

\section{Introduction}

As a hydrophilic and soluble marine polysaccharide macromolecule [1,2], sodium alginate (NaAlg) can form a cross-linked network by interacting with multivalent cations [3] and improve their water and mechanical resistance, barrier properties, cohesion and rigidity [4-6]. Due to its biocompatibility, non-toxicity [7], low cost, and the ability to achieve functional gel through the addition of polyvalent cations, NaAlg has been developed for many applicable purposes [8-12].

Reduced graphene oxide (rGO) is a high-temperature resistant and environmentally friendly material with a melting point of over $4000 \mathrm{~K}$ [13]. It shows important application prospects in advanced materials science [14], new energy and biomedicine, and is considered to be a revolutionary material [15] for the future, making it a current research hotspot. Huang et al. prepared polyvinyl alcohol/rGO nanocomposites by a solution blending method, and the results showed that the composite materials burned to form a dense and uniform carbon layer, which can prevent the thermal mass transfer and exchange of the combustible gas between the matrix material and the outside [16]. Han et al. reported the preparation of the polystyrene/rGO nanocomposites by melt blending and demonstrated that rGO can markedly improve the thermal stability and flame retardant of the polystyrene matrix [17]. And it was demonstrated that the oxides of rGO can improve the mechanical and thermal properties of the matrix materials NaAlg [18]. Furthermore, the hydrogels and aerogels used as adsorbents for ciprofloxacin in wastewater were prepared by Yu et al. by introducing the oxides of rGO into NaAlg, and it was found that the weight loss of the composites was significantly 
lower, compared with that of pure NaAlg [19]. The results of the aforementioned studies suggest that rGO and its oxides can improve the thermal stability of the matrix material NaAlg.

As far as we know, there has been no report regarding the effect of rGO on the thermal stability of calcium alginate (CaAlg). rGO exhibits the characteristic of excellent thermal stability. While the CaAlg formed by cross-linking with $\mathrm{Ca}^{2+}$ based on NaAlg has been reported to be a kind of inherent flame-retardant material, the glycosidic bonds are still easily broken at high temperature, leading to the poor thermal stability. Thus, in this study, rGO was introduced to CaAlg, and the $\mathrm{CaAlg} / \mathrm{rGO}$ nanocomposites were prepared by a sol-gel method in a green and simple way. The materials were characterized by Fourier transform infrared spectroscopy (FT-IR), X-ray diffraction (XRD), scanning electron microscope (SEM) and thermal gravimetric analysis (TGA), and the morphology and thermal stability were studied. Moreover, the thermal degradation mechanism of the nanocomposites was proposed based on the experimental data.

\section{Experimental}

\subsection{Materials}

Sodium alginate (biological grade) was purchased from Zhiyuan Chemical Reagent Co. Ltd. (Tianjin, China). Calcium chloride anhydrous (analytical grade) was purchased from Beichen Fangzheng Reagent Factory (Tianjin, China). Reduced graphene oxide (rGO) was provided by the Institute for Graphene Applied Technology Innovation of Qingdao University (Qingdao, China). All chemicals were used as received without any further purification. All solutions were prepared using deionized water.

\subsection{Preparation of the CaAlg}

The preparation process of the CaAlg material is shown in Scheme 1a. NaAlg of $2.5 \mathrm{~g}$ was dissolved into $47.5 \mathrm{~mL}$ deionized water and stirred to form a homogeneous suspension, standing for $12 \mathrm{~h}$ to be de-foamed afterward. Then $300 \mathrm{~mL}$ of $3 \% \mathrm{CaCl}_{2}$ solution was prepared. Subsequently, the NaAlg suspension was molded by a square mold, and poured into $3 \% \mathrm{CaCl}_{2}$ solution and crosslinked for $24 \mathrm{~h}$ to be solidified. Finally, the solution was washed several times with deionized water and then placed into a drying oven (DHG-9070A, Yuhua Instrument Co., Ltd., Gongyi, China) at $80^{\circ} \mathrm{C}$ to dry to a constant weight.

(a)

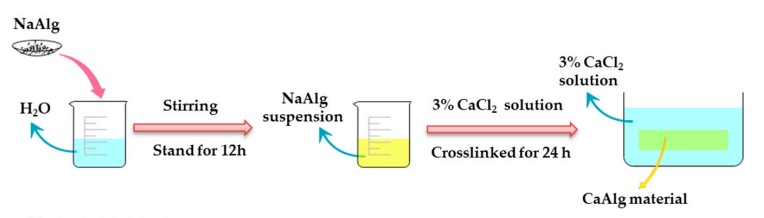

(b)
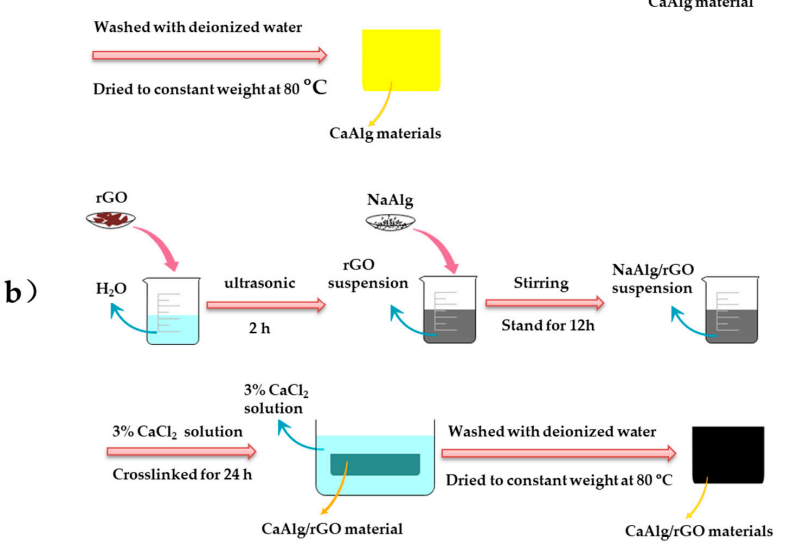

Scheme 1. Schematic diagram of the preparation process of the (a) Calcium alginate (CaAlg) and (b) Calcium alginate/ Reduced graphene oxide (CaAlg/rGO) materials. 


\subsection{Preparation of the CaAlg/rGO Nanocomposites}

The preparation process of the CaAlg/rGO material is shown in Scheme 1b. rGO of $50 \mathrm{mg}$ was added into $47.5 \mathrm{~mL}$ deionized water with ultrasonic bath (KUOS, Kedao Supersonic Instrument Co., Ltd., Shanghai, China) for $2 \mathrm{~h}$, and then was dispersed evenly in deionized water. The solution was slowly added with $2.5 \mathrm{~g}$ of NaAlg into a suspension and stirred to be dissolved to form a homogeneously mixed suspension, standing for $12 \mathrm{~h}$ to be de-foamed afterward. Subsequently, $300 \mathrm{~mL}$ of $3 \% \mathrm{CaCl}_{2}$ solution was prepared, and the $\mathrm{NaAlg} / \mathrm{rGO}$ suspension was molded by a square mold. And then it was poured into $3 \% \mathrm{CaCl}_{2}$ solution and crosslinked for $24 \mathrm{~h}$ to be solidified. Finally, the solution was washed several times with deionized water and then placed into the drying oven at $80^{\circ} \mathrm{C}$ to be dried to a constant weight.

\subsection{Characterizations}

\subsubsection{FT-IR}

FT-IR (NICOLET iS50, Thermo Fisher Scientific Inc., Waltham, MA, USA) was recorded to confirm the chemical characteristics within the wavenumber range of $4000-500 \mathrm{~cm}^{-1}$.

\subsubsection{XRD}

XRD was studied on an X-ray diffractometer (D/Max-RB, Rigaku Inc., Tokyo, Japan) using Cu-K $\alpha$ radiation at room temperature. The samples were scanned up to $80^{\circ}$ in $2 \theta$ in a continuous mode.

\subsubsection{SEM}

SEM (JSM 6390LV, JEOL Inc., Tokyo, Japan) was employed to study the morphology and microstructure of the materials and their cracking residues. All the sample surfaces were sprayed with gold to prevent them from charging under the electron beam.

\subsubsection{TGA}

TGA was analyzed on a thermogravimetric analyzer (TG 209, NETZSCH Geraetebau GmbH, Selb, Germany) with a heating rate of $10^{\circ} \mathrm{C} / \mathrm{min}$. The prepared $5 \mathrm{mg}$ of sample was determined with a flow rate of $50 \mathrm{~mL} / \mathrm{min}$ from room temperature to $1000{ }^{\circ} \mathrm{C}$ under air and $\mathrm{N}_{2}$ atmosphere, respectively.

\section{Results and Discussion}

\subsection{FT-IR Analysis}

The FT-IR spectra of the CaAlg and CaAlg/rGO materials are shown in Figure 1. The main characteristic absorption peak positions and intensity of CaAlg and $\mathrm{CaAlg} / \mathrm{rGO}$ were basically identical $[20,21]$. The broad and strong peaks at $3425 \mathrm{~cm}^{-1}$ can be ascribed to the stretching vibration absorption peak of $-\mathrm{OH}$ bond. The peaks at $2970 \mathrm{~cm}^{-1}$ corresponded to the stretching vibration absorption peak of $-\mathrm{CH}_{2}$ bond, and the peaks at $1629 \mathrm{~cm}^{-1}$ can be assigned to the asymmetric stretching vibration absorption peak of the -COO bond. The peaks at $1447 \mathrm{~cm}^{-1}$ were the in-plane bending vibration absorption peak of the $-\mathrm{CH}_{2}$ bond, and the peaks at $1045 \mathrm{~cm}^{-1}$ can be attributed to the stretching vibration absorption peak of the $\mathrm{C}-\mathrm{O}-\mathrm{C}$ bond [22]. Furthermore, with the addition of $\mathrm{rGO}$, it can be noticed that the stretching vibration absorption peak of $-\mathrm{OH}$ bond at $3425 \mathrm{~cm}^{-1}$ was obviously broadened and the sharpness was decreased which can be due to the superposition of $-\mathrm{OH}$ on the surface of $\mathrm{rGO}$ and $-\mathrm{OH}$ of $\mathrm{CaAlg}$ molecular chain [23]. In addition, the asymmetric stretching vibration absorption peak of $-\mathrm{COO}$ bond at $1629 \mathrm{~cm}^{-1}$ was also broadened, caused by the superposition of $-\mathrm{COO}$ on the rGO surface and $-\mathrm{COO}$ on the $\mathrm{CaAlg}$ molecular chain. The oxygen-containing functional groups, such as $-\mathrm{OH}$ and - $\mathrm{COO}$ on the surface of rGO formed an interfacial hydrogen bond with the CaAlg molecular chain, which can induce close adhesion on the CaAlg and rGO surfaces. Moreover, each carbon atom in $\mathrm{rGO}$ was $\mathrm{sp}^{2}$ hybridized and contributed to the electrons in the remaining one $\mathrm{p}$ 
orbital to form the delocalized $\pi$ bond. The $\pi$ electrons can move freely, while the $-\mathrm{OH}$ of the $\mathrm{CaAlg}$ surface exhibited an electron-donating effect and can enhance the $\pi$-electron cloud density of the conjugated system, thereby inducing a close adhesion of rGO on the CaAlg surface.

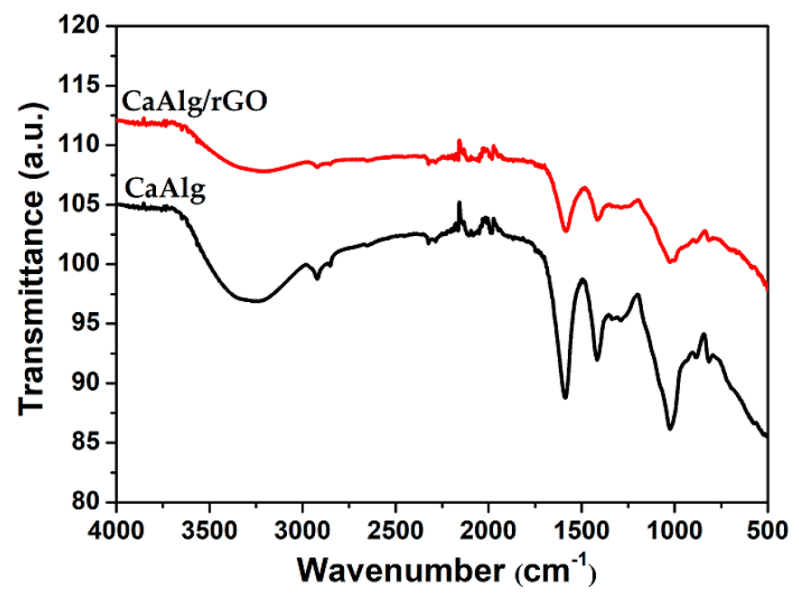

Figure 1. Fourier transform infrared spectroscopy (FT-IR) spectra of the CaAlg and CaAlg/rGO materials.

\subsection{XRD Analysis}

Figure 2 displays the XRD patterns of the CaAlg and CaAlg/rGO materials. As seen, two major diffraction peaks were observed in the CaAlg, namely a weak diffraction peak around $13.3^{\circ}$ and a diffracted peak between $20^{\circ}$ and $30^{\circ}$, while the diffraction peak of rGO was observed at $23.5^{\circ}[24,25]$. It can be noticed from Figure $2 \mathrm{a}$ that the $\mathrm{CaAlg}$ was a high molecular material with certain crystallinity, and its cleavage product at $250{ }^{\circ} \mathrm{C}$ was non-crystalline material, while the ones at $500{ }^{\circ} \mathrm{C}$ were $\mathrm{CaCO}_{3}$ and $\mathrm{Ca}(\mathrm{OH})_{2}$, and the ones at $900^{\circ} \mathrm{C}$ were $\mathrm{CaCO}_{3}, \mathrm{Ca}(\mathrm{OH})_{2}$, and $\mathrm{CaO}$, respectively. Notably, as seen in Figure $3 \mathrm{~b}$, the addition of rGO made the peak of $\mathrm{CaAlg}$ around $13^{\circ}$ and $23^{\circ}$ sharp and strong. Moreover, the characteristic peaks of residual carbon and $\mathrm{CaCO}_{3}$ in the cleavage product can be observed at $250{ }^{\circ} \mathrm{C}$, and the peaks of $\mathrm{CaCO}_{3}$ and $\mathrm{Ca}(\mathrm{OH})_{2}$ at $500{ }^{\circ} \mathrm{C}$, while the peaks of $\mathrm{CaCO}_{3}, \mathrm{Ca}(\mathrm{OH})_{2}$, and $\mathrm{CaO}$ were seen at $900^{\circ} \mathrm{C}$, respectively.
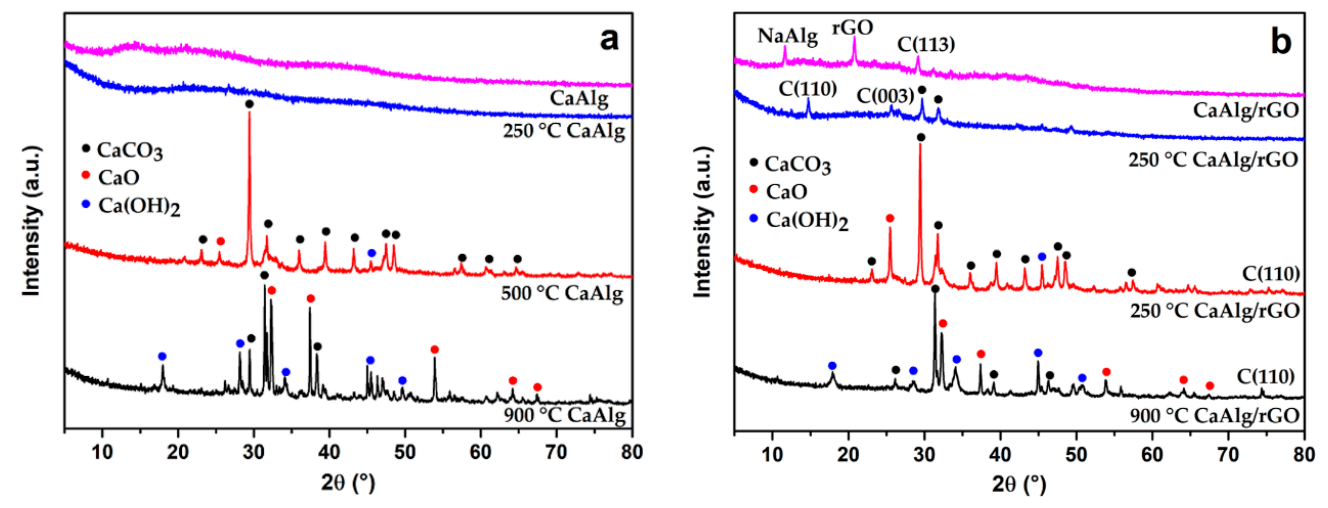

Figure 2. X-ray diffraction (XRD) patterns of the (a) CaAlg and (b) CaAlg/rGO nanocomposites. 


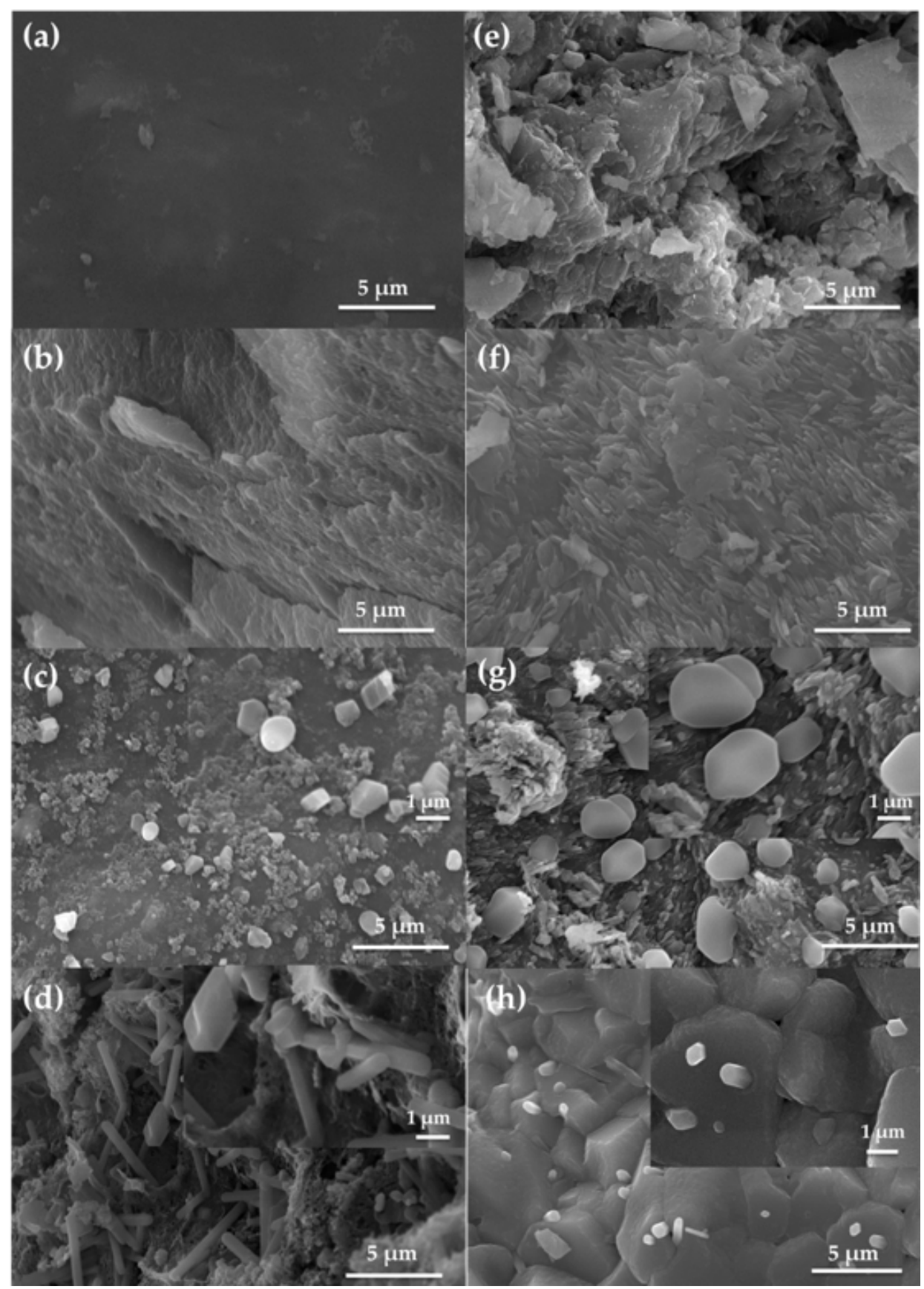

Figure 3. Scanning electron microscope (SEM) images of (a) CaAlg, (b) $\mathrm{CaAlg}$ at $250{ }^{\circ} \mathrm{C}$, (c) $\mathrm{CaAlg}$ at $500{ }^{\circ} \mathrm{C}$, (d) $\mathrm{CaAlg}$ at $900{ }^{\circ} \mathrm{C}$; (e) $\mathrm{CaAlg} / \mathrm{rGO}$, (f) $\mathrm{CaAlg} / \mathrm{rGO}$ at $250{ }^{\circ} \mathrm{C}$, (g) $\mathrm{CaAlg} / \mathrm{rGO}$ at $500{ }^{\circ} \mathrm{C}$, and (h) $\mathrm{CaAlg} / \mathrm{rGO}$ at $900{ }^{\circ} \mathrm{C}$.

\subsection{SEM Analysis}

SEM images of the CaAlg and CaAlg/rGO materials are shown in Figure 3. As seen from Figure $3 \mathrm{a}$,e, the surface of the CaAlg was relatively smooth, and it became rough with the addition of $\mathrm{rGO}$, which may be in the reason that the slices of $\mathrm{rGO}$ were intercalated into the CaAlg [26,27]. As shown in Figure $3 \mathrm{~b}, \mathrm{f}$, the surfaces of the two materials became rough and loose at $250{ }^{\circ} \mathrm{C}$. However, the change of the CaAlg surface was not notable with the introduction of $\mathrm{rGO}$, indicating its role in improving the thermal stability of the CaAlg. As seen from Figure $3 c, g$, many sphere-like crystals, as well as a small amount of irregular spherical crystals and square crystals were observed at $500{ }^{\circ} \mathrm{C}$, 
while the diffraction peak of $\mathrm{CaCO}_{3}, \mathrm{Ca}(\mathrm{OH})_{2}$, and $\mathrm{CaO}$ can be seen from the XRD results. The results suggest that the CaAlg was decomposed into $\mathrm{CaCO} 3$ and $\mathrm{Ca}(\mathrm{OH})_{2}$ at $500{ }^{\circ} \mathrm{C}$ with the generation of little $\mathrm{CaO}$. At $900{ }^{\circ} \mathrm{C}$, lots of clustered spherical crystals, some square crystals and rod-like crystals intercalated on the top, as observed in Figure 3d. According to the diffraction peak in Figure 2a, it can be speculated that the clustered spherical crystals were $\mathrm{CaO}$, and the square crystals were $\mathrm{CaCO}_{3}$, while the rod-like crystals were $\mathrm{Ca}(\mathrm{OH})_{2}$.

Furthermore, it can be observed from Figure $3 \mathrm{~h}$ that the cracking products were relatively well-ordered square crystals, while the embedded spherical crystals and few rod-like crystals can be noticed, as the CaAlg was introduced with rGO and calcined at $900{ }^{\circ} \mathrm{C}$. Combining the XRD patterns shown in Figure $2 b$, it can be inferred that the orderly arranged square crystals should be $\mathrm{CaCO}_{3}$, while the rod-like ones were $\mathrm{Ca}(\mathrm{OH})_{2}$ and spherical ones were $\mathrm{CaO}$, suggesting that $\mathrm{CaCO}_{3}$ in the composites was not decomposed into $\mathrm{CaO}$ even at high temperature. Furthermore, it can be speculated that the carbon formation of the rGO-CaAlg system can cover the surface of the material, and block the thermal mass transfer and the exchange of the combustible gas between the matrix material and the outside. Thus, it may well play a protective role for the matrix material, and improve its thermal stability.

\subsection{TGA Analysis}

\subsubsection{Thermal Degradation Behaviors under $\mathrm{N}_{2}$ Atmosphere}

The thermal stability of the prepared materials under an $\mathrm{N}_{2}$ environment is shown in Figure 4 . As seen from Figure 4a, the curve of the CaAlg/rGO ran above the CaAlg since heating, which showed less thermal degradation than CaAlg did, indicating better thermal stability.
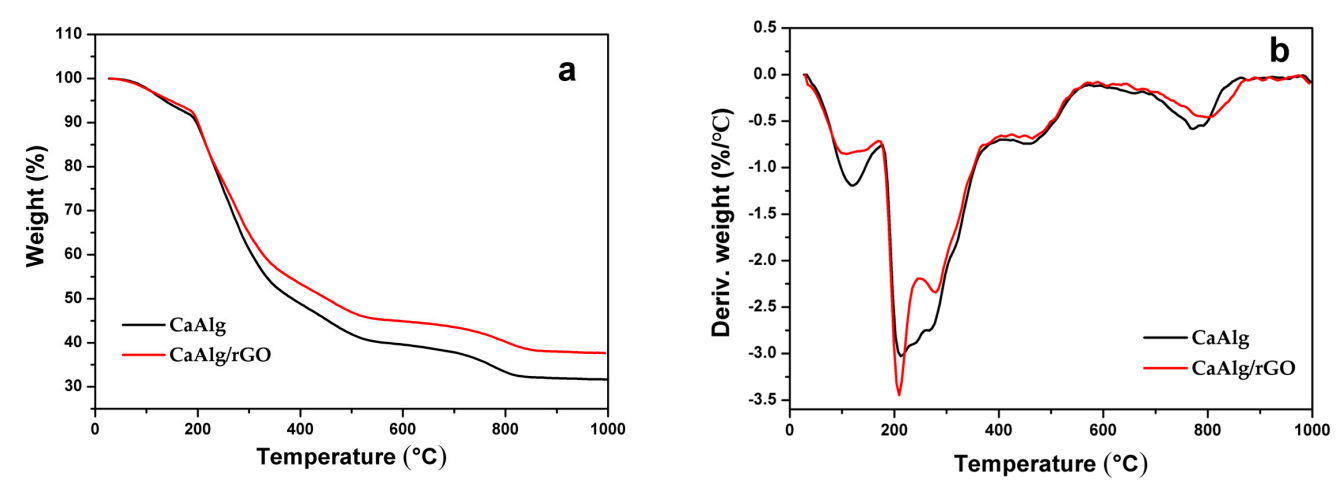

Figure 4. (a) Thermal gravimetric analysis (TGA) and (b) differential thermogravimetric analysis (DTG) of the CaAlg and CaAlg/rGO materials under $\mathrm{N}_{2}$ atmosphere.

As shown in Figure $4 \mathrm{~b}$, the curve of the $\mathrm{CaAlg} / \mathrm{rGO}$ was above the $\mathrm{CaAlg}$ at the beginning of heating, indicating that the thermal degradation rate of the $\mathrm{CaAlg} / \mathrm{rGO}$ was lower than that of the CaAlg. At about $80^{\circ} \mathrm{C}$, both of the materials began to exhibit the first weight loss, while CaAlg/rGO showed a peak weight loss of approximately $3 \%$ at $100{ }^{\circ} \mathrm{C}$. The weight loss peak of the CaAlg lagged behind the CaAlg/rGO slightly, and the peak weight loss around $110{ }^{\circ} \mathrm{C}$ was about $4 \%$. Between $100{ }^{\circ} \mathrm{C}$ and $150{ }^{\circ} \mathrm{C}$, the weight loss peak of the CaAlg was sharper than that of the $\mathrm{CaAlg} / \mathrm{rGO}$, suggesting its moisture content was higher than that of the CaAlg/rGO. Thus it can be inferred that the $\mathrm{CaAlg} / \mathrm{rGO}$ absorbed less water than the CaAlg did, indicating that the intervention of rGO reduced the polarity and hygroscopicity of the CaAlg. At $150{ }^{\circ} \mathrm{C}$, the two materials began to lose weight for the second time. The weight loss peak of the $\mathrm{CaAlg} / \mathrm{rGO}$ was sharper than that of the CaAlg, suggesting a higher weight loss rate of the $\mathrm{CaAlg} / \mathrm{rGO}$, compared to that of the CaAlg. And at $250{ }^{\circ} \mathrm{C}$, the $\mathrm{CaAlg} / \mathrm{rGO}$ continued to be decomposed, with a peak weight loss of $30 \%$ at $280{ }^{\circ} \mathrm{C}$, whereas the CaAlg was only $20 \%$ at $220^{\circ} \mathrm{C}$. This was because after adding rGO, the thermal conductivity of the CaAlg was 
improved, and the heat transfer rate was fast, while the decomposition rate of CaAlg in the material was accelerated. As a result, the weight loss rate of the CaAlg/rGO was higher than that of the CaAlg between $150{ }^{\circ} \mathrm{C}$ and $400{ }^{\circ} \mathrm{C}$. However, the thermal weight loss of the $\mathrm{CaAlg} / \mathrm{rGO}$ was still lower than that of the $\mathrm{CaAlg}$, indicating that rGO can promote the carbon formation of the CaAlg.

At $400-600{ }^{\circ} \mathrm{C}$, the weight loss rates of the $\mathrm{rGO} / \mathrm{CaAlg}$ and CaAlg remained basically the same. Moreover, the weight losses of the two materials also occurred at $600{ }^{\circ} \mathrm{C}$. The weight loss rate of the CaAlg, which was $65 \%$ at $780{ }^{\circ} \mathrm{C}$, was higher than that of the CaAlg/rGO. The weight loss peak of the $\mathrm{CaAlg} / \mathrm{rGO}$ lagged slightly, and a weight loss of $60 \%$ occurred at $800{ }^{\circ} \mathrm{C}$. After the degradation reaction was finished, the curve remained unchanged. Thus, it can be speculated the degradation mechanisms of the $\mathrm{CaAlg} / \mathrm{rGO}$ and $\mathrm{CaAlg}$ were different. Considering thermal stability, the weight loss of the $\mathrm{CaAlg} / \mathrm{rGO}$ at $300-800{ }^{\circ} \mathrm{C}$ was apparently lower than that of the CaAlg, while the weight loss peak of which at $800{ }^{\circ} \mathrm{C}$ remarkably lagged behind that of the CaAlg. Therefore, with the addition of rGO, the thermal stability of the CaAlg was significantly improved.

\subsubsection{Thermal-Oxidative Degradation Behaviors under Air Atmosphere}

Figure 5 shows the TGA results under air atmosphere of the two materials. The thermal performances were similar to that under $\mathrm{N}_{2}$. It can be observed from Figure $5 \mathrm{a}$ that the curve of the $\mathrm{CaAlg} / \mathrm{rGO}$ directly went above the CaAlg after heating, indicating that the CaAlg/rGO possessed better thermal stability than that of the CaAlg. However, as seen in Figure $5 b$, at $200{ }^{\circ} \mathrm{C}$, the $\mathrm{CaAlg} / \mathrm{rGO}$ exhibited a peak weight loss of $26 \%$. Over $250{ }^{\circ} \mathrm{C}$, the CaAlg/rGO continued to decompose with a $36 \%$ of weight loss at $280{ }^{\circ} \mathrm{C}$. And the CaAlg showed a $27 \%$ weight loss peak at $200{ }^{\circ} \mathrm{C}$, and it continued to be decomposed at $250{ }^{\circ} \mathrm{C}$, and its peak weight loss at $290^{\circ} \mathrm{C}$ was $40 \%$. This was because after adding rGO, the thermal conductivity of the material was improved.
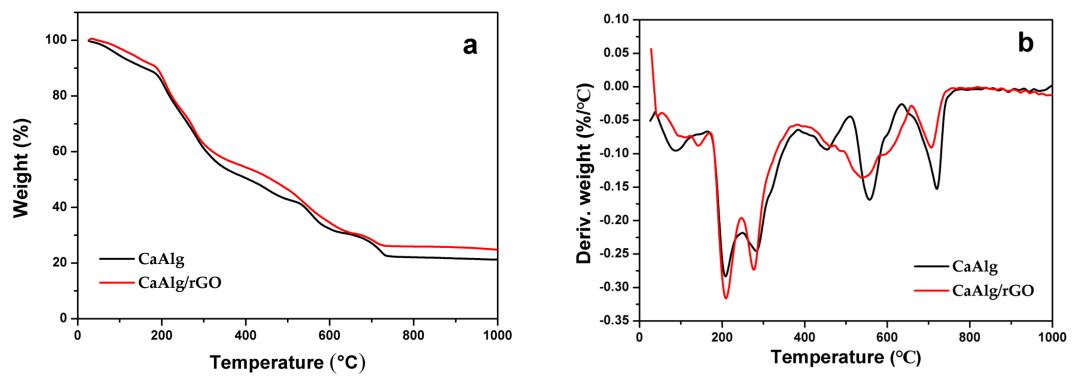

Figure 5. (a) TGA and (b) DTG of CaAlg and CaAlg/rGO materials under air atmosphere.

As a result, the weight loss rate of the CaAlg/rGO was higher than that of the CaAlg between $150{ }^{\circ} \mathrm{C}$ and $400{ }^{\circ} \mathrm{C}$. However, the thermal weight loss of the $\mathrm{CaAlg} / \mathrm{rGO}$ was still lower than that of the $\mathrm{CaAlg}$, indicating that $\mathrm{rGO}$ promoted the carbon formation of the CaAlg. At $450{ }^{\circ} \mathrm{C}$, the $\mathrm{CaAlg}$ showed a notable weight loss peak of $56 \%$, while the peak of the CaAlg/rGO was about $50 \%$. As the two materials continued to be burnt, remarkable weight loss peaks were observed at $540{ }^{\circ} \mathrm{C}$. At $720{ }^{\circ} \mathrm{C}$, the two materials exhibited weight loss again, and the weight loss rate of $\mathrm{CaAlg}$ was higher than that of the $\mathrm{CaAlg} / \mathrm{rGO}$. After the degradation reaction was over, the curve remained almost unchanged.

\subsubsection{Thermal Degradation Mechanisms}

Based on the thermal cracking products of the two materials under $\mathrm{N}_{2}$ and air atmosphere, the thermal degradation mechanism was supposed and displayed in Figure 6, which is further discussed as follows: 


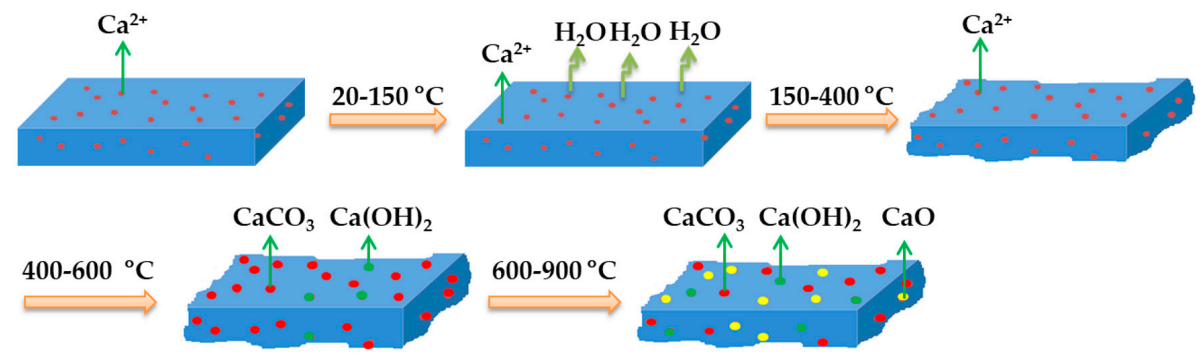

Figure 6. Schematic of the supposed thermal degradation mechanisms.

1. The first stage of thermal degradation

First, the crystal water in the materials was lost at about $120^{\circ} \mathrm{C}$. At $120^{\circ} \mathrm{C}$, as shown in Table 1 , the weight loss rate of the CaAlg was 3.9\% while the $\mathrm{CaAlg} / \mathrm{rGO}$ was $3.7 \%$. It can be seen that the moisture content of the CaAlg was slightly higher, compared with that of the CaAlg/rGO. This shows that, when the moisture regain happened at room temperature, the $\mathrm{CaAlg} / \mathrm{rGO}$ absorbed less water than the CaAlg did, suggesting that the intervention of rGO can reduce the polarity and hygroscopicity of the CaAlg. Both materials released gas water molecules in the early stage of combustion, which can dilute the air and, thus, reduce the burning rate.

Table 1. Comparison of weight losses of Calcium alginate (CaAlg) and Calcium alginate/Reduced graphene oxide (CaAlg/rGO) materials under $\mathrm{N}_{2}$ atmosphere before $200{ }^{\circ} \mathrm{C}$.

\begin{tabular}{cccc}
\hline Parameters & CaAlg & CaAlg/rGO & D-Value \\
\hline Residues at $60^{\circ} \mathrm{C}$ & 99.6 & 99.3 & 0.03 \\
Residues at $90^{\circ} \mathrm{C}$ & 98.3 & 98.0 & 0.03 \\
Residues at $120^{\circ} \mathrm{C}$ & 96.1 & 96.3 & 0.02 \\
Residues at $150{ }^{\circ} \mathrm{C}$ & 93.8 & 94.6 & 0.08 \\
Residues at $180^{\circ} \mathrm{C}$ & 92.0 & 92.9 & 0.09 \\
\hline
\end{tabular}

2. The second stage of thermal degradation

As seen from TG curves (Figures 4 and 5), the decomposition temperature of the CaAlg was about $250{ }^{\circ} \mathrm{C}$. It can be observed from Table 2 that, at $250{ }^{\circ} \mathrm{C}$, the weight loss rate of the CaAlg was $26.0 \%$ while the $\mathrm{CaAlg} / \mathrm{rGO}$ was $24.0 \%$, between which the difference was only $2.0 \%$. As the amount of rGO added to the composites was $2.0 \%$, thus rGO did not notably promote the carbon formation of the CaAlg at $250{ }^{\circ} \mathrm{C}$. At $450{ }^{\circ} \mathrm{C}$, the difference of the weight loss rate between the CaAlg and the CaAlg/rGO was $4.6 \%$, indicating the carbonization rate of the composites increased by $2.6 \%$. Up to $750{ }^{\circ} \mathrm{C}$, the difference of the weight loss rose to $6.1 \%$, and the carbonization rate reached $4.1 \%$. The carbonization rate of the two materials did not change anymore when the temperature was higher than $850{ }^{\circ} \mathrm{C}$. It can be inferred that introducing $\mathrm{rGO}$ to the $\mathrm{CaAlg}$ matrix can promote the rate carbonization, contributing to the enhanced thermal stability of the $\mathrm{CaAlg} / \mathrm{rGO}$, compared with that of the CaAlg. It shows that $\mathrm{rGO}$ can not only form carbon itself but also promote the carbon formation of the CaAlg. The formed carbon layer covered the surface of the materials, which blocked the thermal mass transfer and the exchange of the combustible gas between the materials and the outside. It can play a great protective role for the matrix material, and effectively improve its thermal stability. At this time, $\mathrm{CO}_{2}$ played the gas phase role, while the carbon formation of the rGO-CaAlg system, the endothermic decomposition of $\mathrm{CaCO}_{3}$ and the cover effect of $\mathrm{CaO}$ played the solid phase role together. It can be seen that the formation of the rGO-CaAlg system led to a change in the thermal degradation behavior of the CaAlg, as shown in Scheme 2. 
Table 2. Comparison of the weight losses of the CaAlg and CaAlg/rGO materials under $\mathrm{N}_{2}$ atmosphere over $200^{\circ} \mathrm{C}$.

\begin{tabular}{ccccc}
\hline Paremeters & CaAlg & CaAlg/rGO & D-Value & Carbonization Rate \\
\hline Residues at $250^{\circ} \mathrm{C}$ & 74.0 & 76.0 & 2.0 & 0 \\
Residues at $350^{\circ} \mathrm{C}$ & 52.7 & 56.8 & 4.1 & $2.1 \%$ \\
Residues at $450^{\circ} \mathrm{C}$ & 45.2 & 49.8 & 4.6 & $2.6 \%$ \\
Residues at $550{ }^{\circ} \mathrm{C}$ & 40.2 & 45.3 & 5.1 & $3.1 \%$ \\
Residues at $650{ }^{\circ} \mathrm{C}$ & 38.8 & 44.3 & 5.5 & $3.5 \%$ \\
Residues at $750^{\circ} \mathrm{C}$ & 36.0 & 42.1 & 6.1 & $4.1 \%$ \\
Residues at $850^{\circ} \mathrm{C}$ & 32.2 & 38.3 & 6.1 & $4.1 \%$ \\
\hline
\end{tabular}

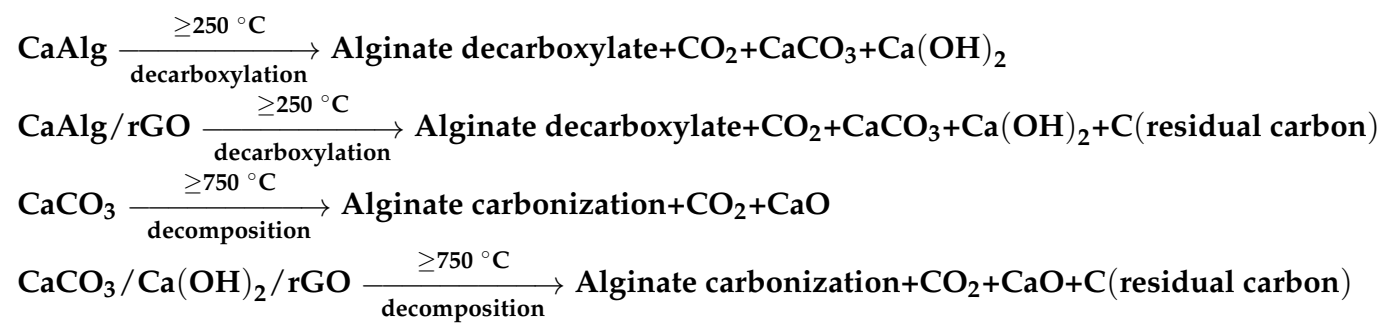

Scheme 2. The schematic of the thermal degradation reactions of the CaAlg and CaAlg/rGO.

The two materials can provide the ionization of carboxyl groups since they exhibited alkaline properties in the thermal cracking process, and, thus, the decarboxylation reaction of polysaccharide chains for the CaAlg was promoted while heating. Further description of the reaction is shown in Scheme 3, in which R represents the glycoside fragments generated from the decarboxylation of the macromolecular glycan.

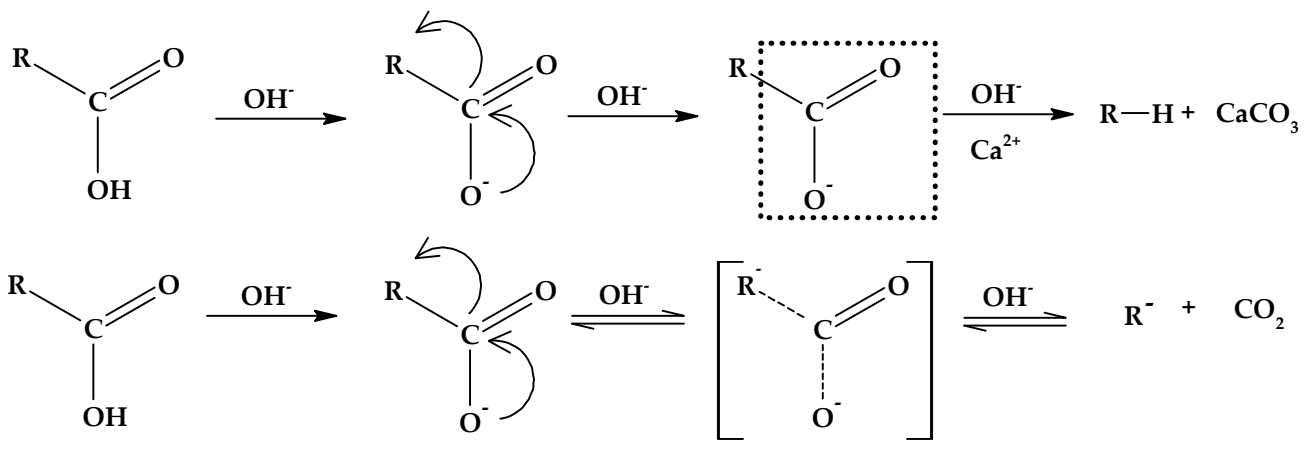

Scheme 3. The schematic of decarboxylation mechanisms of the CaAlg and CaAlg/rGO materials.

\section{Conclusions}

The CaAlg/rGO nanocomposites were fabricated through introducing rGO into the CaAlg by a green and facile sol-gel method, and their morphological and thermal properties were studied. Moreover, the underlying mechanisms of thermal degradation were supposed based on the experimental data. It was found that after the addition of $\mathrm{rGO}$, a synergistic system of rGO-CaAlg was formed, and the thermal stability of the system was remarkably improved. The introduction of rGO promoted the formation of carbon from the CaAlg, and the carbonization rate reached $4.1 \%$. The formed carbon layer covered the surface of the substrate and blocked the thermal mass transfer and the exchange of the combustible gas between the matrix material and the outside. It played a very good protective role for the matrix material and improved its thermal stability. Furthermore, the prepared 
$\mathrm{CaAlg} / \mathrm{rGO}$ composites are biodegradable and eco-friendly materials. Therefore, the materials can be promising when applied to the industry of new textile, heating, and flame retardant materials.

Author Contributions: Q.L. and Z.L. conceived and designed the experiments; W.Z. and P.X. performed the experiments; Y.Q., Y.W., Y.X. and Q.L. analyzed the data; W.Z. and Z.L. wrote the paper.

Funding: This work was funded by the National Natural Science Foundation of China (grant number 51773102, 51503110), and Natural Science Foundation of Shandong Province (grant number ZR2014JL010).

Conflicts of Interest: The authors declare no conflict of interest.

\section{References}

1. Yang, J.S.; Xie, Y.J.; He, W. Research progress on chemical modification of alginate: A review. Carbohydr. Polym. 2011, 84, 33-39. [CrossRef]

2. Algothmi, W.M.; Bandaru, N.M.; Yu, Y.; Shapter, J.G.; Ellis, A.V. Alginate-graphene oxide hybrid gel beads: An efficient copper adsorbent material. J. Colloid Interface Sci. 2013, 397, 32-38. [CrossRef] [PubMed]

3. Yang, C.H.; Wang, M.X.; Haider, H.; Yang, J.H.; Sun, J.Y.; Chen, Y.M.; Zhou, J.; Suo, Z. Strengthening alginate/polyacrylamide hydrogels using various multivalent cations. ACS Appl. Mater. Interfaces 2013, 5, 10418-10422. [CrossRef] [PubMed]

4. Zheng, H.; Yang, J.; Han, S. The synthesis and characteristics of sodium alginate/graphene oxide composite films crosslinked with multivalent cations. J. Appl. Polym. Sci. 2016, 133. [CrossRef]

5. Crossingham, Y.J.; Kerr, P.G.; Kennedy, R.A. Comparison of selected physico-chemical properties of calcium alginate films prepared by two different methods. Int. J. Pharm. 2014, 473, 259-269. [CrossRef] [PubMed]

6. Kilan, K.; Warszyński, P. Thickness and permeability of multilayers containing alginate cross-linked by calcium ions. Electrochim. Acta 2014, 144, 254-262. [CrossRef]

7. Liu, Y.; Chen, S.; Zhong, L.; Wu, G. Preparation of high-stable silver nanoparticle dispersion by using sodium alginate as a stabilizer under gamma radiation. Radiat. Phys. Chem. 2009, 78, 251-255. [CrossRef]

8. Barrett, P.R.F. Some studies on the use of alginates for the placement and controlled release of diquat on submerged aquatic plants. Pest Manag. Sci. 2010, 9, 425-433. [CrossRef]

9. Kennedy, J.F.; Barnes, J.A.; Barker, S.A. The use of alginate ester films on solid supports in the preparation of water-insoluble immunoadsorbents for purification of antigens. Eur. J. Biochem. 1983, 133, 697-705. [CrossRef] [PubMed]

10. Sikareepaisan, P.; Ruktanonchai, U.; Supaphol, P. Preparation and characterization of asiaticoside-loaded alginate films and their potential for use as effectual wound dressings. Carbohydr. Polym. 2011, 83, 1457-1469. [CrossRef]

11. Li, J.; Li, Z.; Zhao, X.; Deng, Y.; Xue, Y.; Li, Q. Flame retardancy and thermal degradation mechanism of calcium alginate/caco3 composites prepared via in situ method. J. Therm. Anal. Calorim. 2017, 131, 2167-2177. [CrossRef]

12. Liu, Z.; Li, Z.; Zhao, X.; Zhang, L.; Li, Q. Highly efficient flame retardant hybrid composites based on calcium alginate/nano-calcium borate. Polymers 2018, 10, 625. [CrossRef]

13. Los, J.H.; Zakharchenko, K.V.; Katsnelson, M.I.; Fasolino, A. Melting temperature of graphene. Phys. Rev. B 2015, 91, 404-406. [CrossRef]

14. Guo, T.; Zhang, G.; Su, X.; Zhang, H.; Wan, J.; Chen, X.; Wu, H.; Liu, C. Transparent and flexible capacitors with an ultrathin structure by using graphene as bottom electrodes. Nanomaterials 2017, 7, 418. [CrossRef] [PubMed]

15. Celik, N.; Manivannan, N.; Strudwick, A.; Balachandran, W. Graphene-enabled electrodes for electrocardiogram monitoring. Nanomaterials 2016, 6, 156. [CrossRef] [PubMed]

16. Huang, G.; Gao, J.; Wang, X.; Liang, H.; Ge, C. How can graphene reduce the flammability of polymer nanocomposites? Mater. Lett. 2012, 66, 187-189. [CrossRef]

17. Han, Y.; Wu, Y.; Shen, M.; Huang, X.; Zhu, J.; Zhang, X. Preparation and properties of polystyrene nanocomposites with graphite oxide and graphene as flame retardants. J. Mater. Sci. Lett. 2013, 48, 4214-4222. [CrossRef]

18. Ionita, M.; Pandele, M.A.; Iovu, H. Sodium alginate/graphene oxide composite films with enhanced thermal and mechanical properties. Carbohydr. Polym. 2013, 94, 339. [CrossRef] [PubMed] 
19. Fei, Y.; Li, Y.; Han, S.; Ma, J. Adsorptive removal of ciprofloxacin by sodium alginate/graphene oxide composite beads from aqueous solution. J. Colloid Interface Sci. 2016, 484, 196-204. [CrossRef] [PubMed]

20. Pandi, K.; Viswanathan, N. A facile synthesis of metal ion-imprinted graphene oxide/alginate hybrid biopolymeric beads for enhanced fluoride sorption. RSC Adv. 2016, 6, 75905-75915. [CrossRef]

21. Shu, B.; Wu, S.; Dong, L.; Wang, Q.; Liu, Q. Microfluidic synthesis of ca-alginate microcapsules for self-healing of bituminous binder. Materials 2018, 11, 630. [CrossRef] [PubMed]

22. Luo, Y.; Lode, A.; Wu, C.; Jiang, C.; Gelinsky, M. Alginate/nanohydroxyapatite scaffolds with designed core/shell structures fabricated by $3 \mathrm{~d}$ plotting and in situ mineralization for bone tissue engineering. ACS Appl. Mater. Interfaces 2015, 7, 6541-6549. [CrossRef] [PubMed]

23. Ramesha, G.K.; Kumara, A.V.; Muralidhara, H.B.; Sampath, S. Graphene and graphene oxide as effective adsorbents toward anionic and cationic dyes. J. Colloid Interface Sci. 2011, 361, 270-277. [CrossRef] [PubMed]

24. Chen, K.; Ling, Y.; Cao, C.; Li, X.; Chen, X.; Wang, X. Chitosan derivatives/reduced graphene oxide/alginate beads for small-molecule drug delivery. Mater. Sci. Eng. C 2016, 69, 1222-1228. [CrossRef] [PubMed]

25. Abdulkhani, A.; Daliri Sousefi, M.; Ashori, A.; Ebrahimi, G. Preparation and characterization of sodium carboxymethyl cellulose/silk fibroin/graphene oxide nanocomposite films. Polym. Test. 2016, 52, $218-224$. [CrossRef]

26. Jiao, C.; Xiong, J.; Tao, J.; Xu, S.; Zhang, D.; Lin, H.; Chen, Y. Sodium alginate/graphene oxide aerogel with enhanced strength-toughness and its heavy metal adsorption study. Int. J. Biol. Macromol. 2016, 83, $133-141$. [CrossRef] [PubMed]

27. Li, Y.; Du, Q.; Liu, T.; Sun, J.; Wang, Y.; Wu, S.; Wang, Z.; Xia, Y.; Xia, L. Methylene blue adsorption on graphene oxide/calcium alginate composites. Carbohydr. Polym. 2013, 95, 501-507. [CrossRef] [PubMed]

(C) 2018 by the authors. Licensee MDPI, Basel, Switzerland. This article is an open access article distributed under the terms and conditions of the Creative Commons Attribution (CC BY) license (http:/ / creativecommons.org/licenses/by/4.0/). 